\title{
Design and Implementation of Online E-business Website Based on Java
}

\author{
Diao Zhijian
}

Nanchang Institute of Science \& Technology, Nanchang, China, 330108

Keywords: Java Technology, E-business, Website Design

\begin{abstract}
Java technology is a subject-oriented programming language with sound visual editorial environment and performance, playing an important role in establishing e-business website. With the rapid development of Internet technology, the advent of emerging e-business model has transformed the traditional business model to a large degree, realizing efficient, low-cost connection between enterprises and consumers and making convenient and fast consumption. While e-business model is to design and develop online e-business website technologically by making full use of advantages of Java technology, which has great significance for improving feasibility and scientificity of e-business website. Meanwhile, the optimization and development of e-business model can bring better usage experience for enterprises and consumers, exerting positive effect on key targets of design and development of e-business websites. This paper, starting from development procedures of e-business website, analyzes the advantages and features of Java technology, and further discusses the design and implementation of online e-business website based on Java to promote deep development of e-business model in China.
\end{abstract}

\section{Introduction}

The rapid development of e-business model in China over recent years has spread to all aspects of people's life, bringing radical changes for traditional business operating model and consumption model. Online e-business model carries convenience for consumers and enterprises, at the same time, e-business website based on Internet technology and the superiority of Internet technology have directly influenced the operation of e0business websites. As a subject-oriented programming language, Java technology boasts sound visual editorial environment and performance, playing a vital part in building e-business websites. The application of Java technology in design and development of online e-business websites is of great importance for feasibility and scientitificity improvement of e-business websites. At the same time, the optimization and development of e-business model can bring better user experience for enterprises and consumers, which is conducive to reaching goals of design and implementation of e-business websites. How to promote Java technology to play an important part in online e-business making full use of advantages of Java technology has become a hot issue worth thinking and discussing. Starting from development procedures of e-business websites, the paper analyzes the advantages and characteristics of Java technology, deeply discusses the design and implementation of online e-business websites based on Java technology. It studies Java-based online e-business websites to promote the further development of e-business model in China.

\section{Development process of online e-business website}

The design and implementation of online e-business websites based on Internet technology shall obey relevant procedure, which can be divided into five stages. First is preparation, the demand analysis of e-commerce website system. The main goal of this stage is to prepare design and development of e-business websites and know the reason for building e-business websites, the goals of e-business websites and so on. After that, it is necessary to prepare needed data and information. Meanwhile, by investigating user information and demands for software function, the paper analyzes the demand of e-commerce website system and establishes relevant websites fully meeting needs of users to so as to ensure the e-business website to play an important role in practice. Second, 
the demand analysis of user information. The establishment of online e-business website mainly serves for users, so the analysis of user demands to learn user demands for e-business website system as a reference is made to achieve the ultimate goal. Third, induction and reorganization. The major task of this stage is to make a comprehensive analysis of all results collected from first-phase preparations and make main goals of developing e-business website clear. The e-business website development model is optimized to basically define the development direction of e-business websites.

Forth, e-business system design. In this stage, user demands and function demands are analyzed, and problems in development of e-business websites are solved to design corresponding program coding and build all modules. Fifth, system implementation. This is the final stage in design and development of online e-business websites in which systems must be tested to learn problems in operating e-business website systems. The stage will stifle all factors impacting e-business website system operation in the cradle and promote the safe and stable operation of e-business website systems, realizing important targets of design and development of online e-business websites.

\section{Advantages and characteristics of Java Technology}

Like $\mathrm{C}$ language and $\mathrm{C}++$ language in some degree, Java language and $\mathrm{C}++$ language, however, are two totally different languages with unique and distinct advantages, which cannot be replaced or merged. Java is the most-widely-used online programming language at present, simple, stable and dynamic, being of great significance for practical application. The simplicity of Java technology lies in its easy use. Like $\mathrm{C}++$, there are many similar languages in Java such as loop statement, control statement, etc. But it is worth noting that Java cannot be regarded as the same thing as $\mathrm{C}++$. Based on subject-oriented program, Java conforms to human's thinking patterns, realizes internet technology according to actual needs of subjects, which meets the needs of uses widely and plays an important role in practice. After the comparison of Java language, $\mathrm{C}$ language and $\mathrm{C}++$ language, it can be found that the irrelevance to platform is the largest advantage of Java language. The main reason is that Java language can make changes to operating systems which other language programs fail to do. For example, the changes of core system will bring about malfunction of system operation. While Java language can resolve this problem properly, whose system changes with the change of core system and processor to support the operation of new programs and achieve significant goals of system design and development by using Java language. And the safety of Java technology is another important reason for its wide application in various fields. As for the actual application of Java language, its authorization should be set in practice to ensure the safety of interviewees. In light of characteristics of online e-business websites, it is subject-oriented and focuses on the safety of subjects. Given such features, making full use of technological advantages of Java language can really achieve major goals of wide application of Java technology. The easy, simple, safe Java technology irrelevant to platform is an important guarantee for its wide application in all areas. Given the design and development of online e-business websites in this paper, this process relies on relevant Internet technologies. And giving unique advantages of Java technology full play can provide full guarantee for design and development of e-business websites.

\section{Analysis of design and implementation of Java-based online e-business website}

Java technology is a subject-oriented programming language with sound visual editorial environment and performance, playing a vital role in building e-business websites. The reason for Java technology's wide application is its safety, easy operation and irrelevance to platform. Application of Java technology in design and development of online e-business website is of great significance for feasibility and scientificity of e-business websites. Besides, optimization of e-business model can bring better user experience for enterprises and consumers, exerting positive impact on achieving goals of e-business websites. Therefore, how to promote application of Java technology in e-business website design is an issue worth consideration and discussion. At same time, it is necessary to learn procedure of online e-business website design in using scientific 
technology to show value of Java technology.

\subsection{System operating environment}

The safety of system operating environment has a direct connection with the stable operation of online e-business websites as well as effective correlation between consumers and enterprises. As an advanced internet technology, Java technology has advantages of safety, simple operation, which has nothing with platform. So the online e-business website design based on Java technology can offer security for design and development of e-business websites. However, many factors will interfere the operation in the practice. The main part is the direct effect of internet environment on online e-business websites. For instance, the breakdown and collapse of large internet environment will influence the operation of e-business websites. Thus, sate inspection will be conducted in the design and development. Once accident is found, emergency measures shall be launched. The sound system operation environment can provide guarantee for safe and stable operation of online Java-based e-business websites.

\subsection{System function positioning}

The main goal of design and implementation of Java-based online e-business website is to exhibit, sale and deliver physical commodities,

Concentrate products and consumer services, reach maximum of interests, attract more consumers to shop online, bringing more profits for online e-business website users. One prominent strength of online e-business website is the transparency of products, consumption and so on, largely meeting the psychological needs of consumers. In addition, the after-sale service has largely improved user experience. Taking online shopping mall of Taobao as an example, a variety of products are offered to consumers with many options, and after sales, the follow-up transparent transport state can provide better online shopping experience for consumers. While for online e-business website developers, the online e-business website can provide a platform for exhibiting and selling products and guarantee for consumers according to their consumption preferences.

\subsection{User demand analysis}

The major aim of building online e-business websites is to serve for users and analyze demands of users for website system as a reference. As for Java technology, the prominent characteristics is subject-oriented, which conforms to human's thinking pattern. Realizing internet technology in line with actual demands of subjects can meets their needs and play a significant role in Java's practice. Meanwhile, it is noted that the user demands must be met to write programs and provide better user experience.

Only by doing this can important goals of online e-business website system design and development can be achieved.

\subsection{System operator demand analysis}

Online e-business website is to meet the needs of system operators and reach benefit maximization. In applying Java technology in building e-business websites, preparation work must be done and some problems be solved, for example, why build e-business websites?

What are the goals of e-business websites? After solving these problems, it is important to prepare needed data and information. As for the demand analysis of e-business website system, surveys on user information and software function should be made to establish relevant websites which satisfy users more and ensure the important position of e-business website in practical application. Therefore, Java-based online e-business website should take basic demands of system operators into consideration in design, ensuring safe and stable operation of e0business websites.

\section{Conclusion}

In a word, although similarity exits among Java language, $\mathrm{C}$ language and $\mathrm{C}++$ language, the superiority of Java technology lays a solid foundation for its wide application in multiple fields. 
Under the large Internet environment, how to utilize Java technology to design online e-business website has become an issue worth consideration and discussion.

\section{References}

[1] Yu Chaofeng, Xiong Ying, Zhang Yong, Design and implementation of quality extension credit website of Nanchang Hangkong University based on Java. [J]. Shangqing, 2012, (16):15-15.

[2] Li Can. Design and implementation of online booking website of sports halls in universities--taking table tennis gym in Zhengzhou University as an example [J]. Henan Science \& Technology, 2015, (15):10-12.

[3] Chen Guoyao, Research on automatic website data collection and anti-automatic data collection--the implementation of Java programming language based on Windows platform. [J]. Technology Innovation and Application, 2015, (11):102-102.

[4] Wang Rong, Analysis of online HTML text editor in Java web development--taking KindEditor as an example [J]. Journal of Hubei University of Science, 2013, 33(6):21-23.

[5] Xing Qi, Zhao Weidong, Liu Junna, Design and implementation of question-answering system of enterprise portals based on Lucene.[J].Software Guide, 2015,14(7):109-110.

[6] Tang Na, The application and implementation of format design in e-business websites--taking MANFOO cosmetics website as an example [J].West Leather, 2016, (10):33.

[7] zhang Tao. B282C e-commerce model and e-commerce platform based on J2EE design architecture [J]. Journal of Chengdu University: Natural Science, 2012, 31(4):405-407.

[8] Yuan Yujiao, Ye Chunming, Sun Junhua et al. Research on mobile phone shopping web design based on dynamic line analysis and system layout design [J]. Human Ergonomics, 2016, 22(1):32-38, 43.

[9] Yang li, Wen Chunyan. Design of online book sales system based on ASP.NET technology--design of online book sale system [J]. China science and technology information, 2011,(5):95-97.

[10] Chen. Strategic research on reconfiguration planning of e-commerce sites--user-centered design concept [J]. China business, 2012,(15):103-104.

[11] Liu Jing. A brief analysis on cultivating students' innovative ability and comprehensive learning ability -- e-commerce B2C website design [J]. China E-commerce, 2011,(6):2-3.

[12] Guo Weigang, Cen Jianlin, Zhong Yong, etc. Discussion on the construction and operation mode of regional network teaching resource base based on e-commerce concept [J]. China education informationization · basic education, 2010, (5):69-71. 